\title{
Ganglioneuroma retroperitoneal en la infancia
}

\author{
Arce Gil J, Arce Terroba Y*, Angerri Feu O, Caffaratti Sfulcini J, Garat Barredo JMa, \\ Villavicencio Mavrich H.
}

\begin{abstract}
Servicio Urología y Anatomía Patológica*. Fundació Puigvert, Barcelona.
\end{abstract}
Actas Urol Esp. 2008;32(5):567-570

\section{RESUMEN}

GANGLIONEUROMA RETROPERITONEAL EN LA INFANCIA

En la infancia, no existen guías específicas para el manejo de los incidentalomas suprarrenales detectados postnatalmente, pero dada la alta proporción de tumores malignos se recomienda su resección quirúrgica. Presentamos el caso de una paciente de 7 años diagnosticada de un incidentaloma suprarrenal izquierdo no funcionante. La exéresis quirúrgica de dicha masa y el posterior estudio histopatológico confirmaron el diagnóstico de ganglioneuroma en proceso de maduración. La mayoría de ganglioneuromas son incidentales y presentan un buen pronóstico dado su comportamiento benigno, aunque está descrita la transformación maligna, recomendándose realizar controles postoperatorios para detectar las recidivas locales y la aparición de nuevos focos.

Palabras clave: Incidentaloma. Ganglioneuroma. Suprarrenal

\section{ABSTRACT}

RETROPERITONEAL GANGLIONEUROMA IN THE INFANCY

Specific management guides for suprarenal incidentalomas detected after birth do not exist for children, but due to the high proportion of malignant tumors, surgical resection is recommended. We present the case of a 7-yearold patient with a non-functioning left suprarenal incidentaloma. Surgical resection and subsequent anatomopathologic analysis of the mass confirmed the diagnosis of ganglioneuroma in process of maturation. Most ganglioneuromas are incidental and have a good prognosis due to their benign behaviour, even though malignant transformation has also been described, for which reason postoperative controls to detect local recurrence and occurrence of new foci are recommended.

Keywords: Incidentaloma. Ganglioneuroma. Suprarenal

$\mathrm{L}$ as lesiones suprarrenales halladas por métodos de imagen durante el estudio de otras patologías se conocen como "incidentalomas suprarrenales”. Aunque estas lesiones son raras, su incidencia está en aumento dado el incremento en la indicación de pruebas de imagen ${ }^{1}$.

En adultos, la mayor parte de las masas suprarrenales son adenomas habiéndose desarrollado guías de evaluación y tratamiento precisas para el manejo de incidentalomas no funcionantes. En la infancia, el neuroblastoma es la masa adrenal más frecuente, sin que se hayan establecido guías específicas en el manejo de los incidentalomas suprarrenales infantiles ${ }^{2,3}$.

\section{CASO CLÍNICO}

Paciente de 7 años de edad, primera hija producto de un embarazo y parto normal, con un peso al nacimiento de 3250 gramos, diagnosticada de reflujo vésico-ureteral derecho grado III al año de vida tras 3 episodios de ITU febril, que desaparece a los 4 años de edad. En control rutinario se diagnosticó por ecografía (Fig. 1) de una tumoración localizada a nivel suprarrenal izquierda de $6 \times 4 \mathrm{~cm}$ de aspecto sólido y homogéneo. El TAC abdominal (Fig. 2) confirmó la presencia de una masa a nivel suprarrenal izquierdo de $6 \times 4 \mathrm{~cm}$ homogénea y bien delimitada, con microcalcificaciones y densidad de partes blandas que presentaba captación segmentaria 


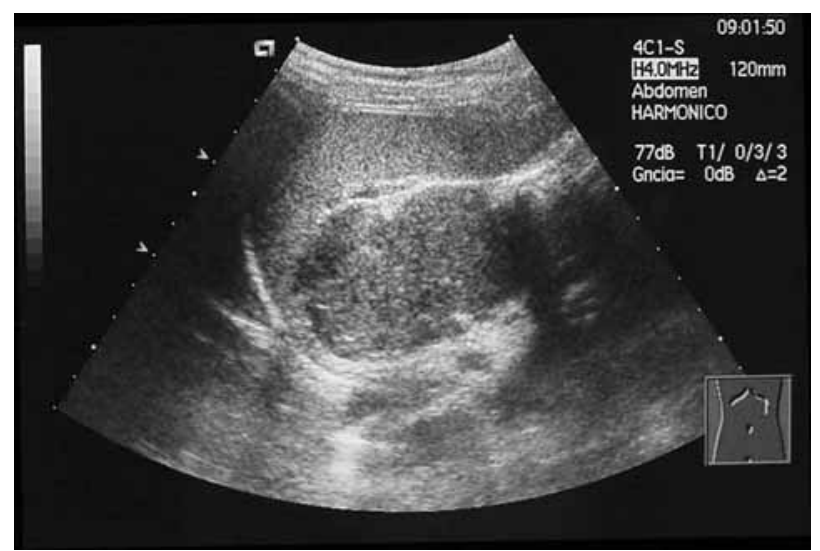

FIGURA 1: Eco reno-vesical. Lesión tumoral en zona suprarrenal izquierda de aspecto sólido y homogéneo.

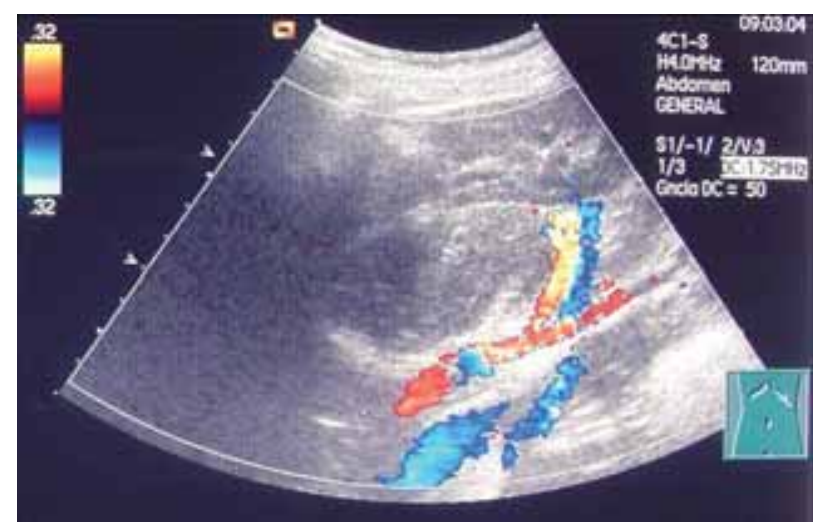

FIGURA 2: Eco doppler color. Lesión tumoral en contacto con arteria y vena renal izquierdas (en colores).

del contraste sin que se apreciaran adenopatías. Las determinaciones de cortisol, aldosterona y metanefrinas en sangre y orina fueron normales.

Ante la aparición incidental de esta masa suprarrenal, y la alta sospecha de neuroblastoma se realizó la exéresis completa de la masa suprarrenal vía laparoscópica. Macroscópicamente (Fig. 3), la tumoración era independiente de la glándula suprarrenal, de forma polilobulada midiendo $7 \times 5 \times 4,4 \mathrm{~cm}$. Presentaba una superficie externa lisa y rojiza mostrando al corte un aspecto homogéneo, blanquecino y firme con áreas fasciculadas sin apreciarse áreas de hemorragia o necrosis. Microscópicamente (Fig. 4), la tumoración tenía unos bordes bien definidos y estaba constituida predominantemente por un estroma neurofibromatoso formado por pequeños fascículos de células de Schwann, identificándose células ganglionares entremezcladas con el estroma, dispuestas individualmente o en pequeños grupos,

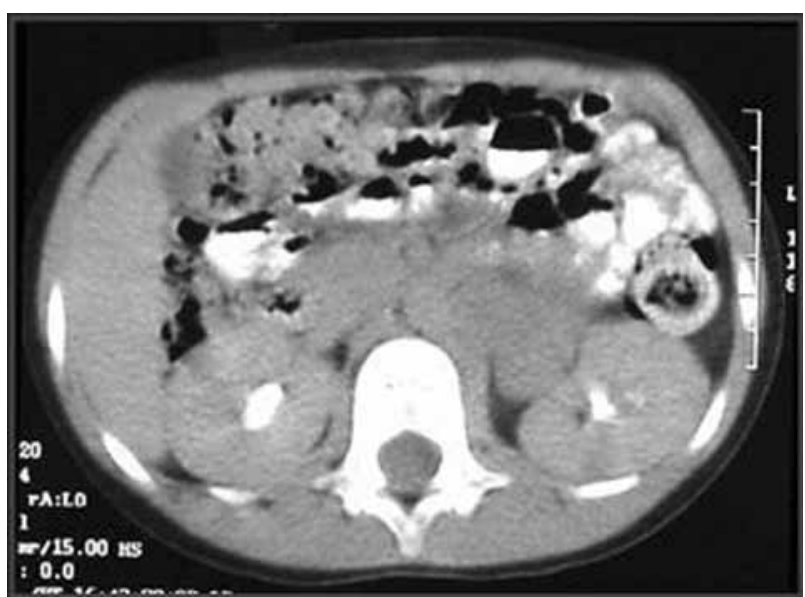

FIGURA 3: TAC abdominal. Tumoración de $6 x 4 \mathrm{~cm}$ homogénea y bien delimitada con microcalcificaciones y densidad de partes blandas. Captación segmentaria del contraste. No adenopatias.

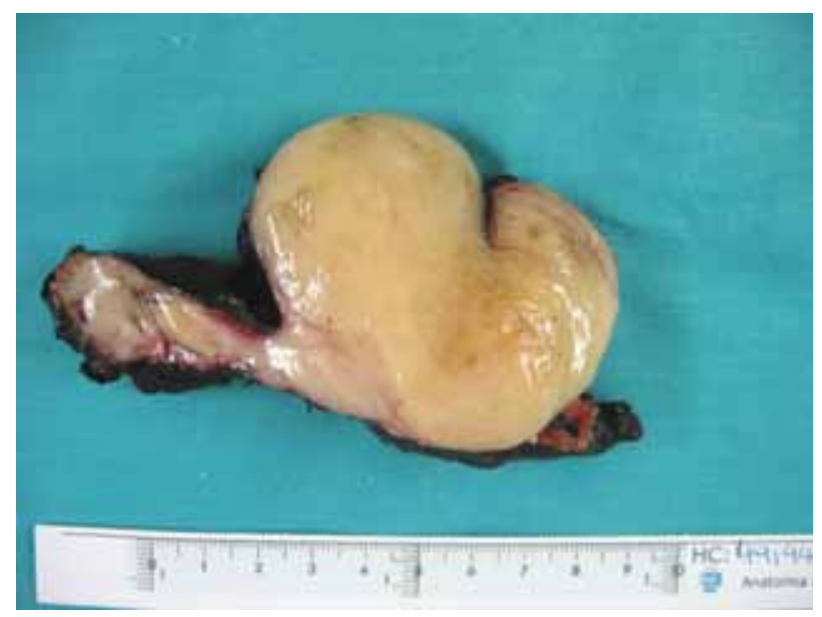

FIGURA 4: a) La masa tumoral está constituida de forma predominante por estroma neurofibromatoso, compuesto por células alargadas que se agrupan en haces con un caracteristico patrón ondulante. b) Células ganglionares entremezcladas con el estroma. c) Áreas de diferenciación tipo neuropilo con presencia de células ganglionares. d) Agregado basófilo e irregular que corresponde a una microcalcificación estromal, caracteristicas en estos tumores.

mostrando algunas de ellas un aspecto inmaduro. Se identificaron microcalcificaciones estromales. Las tinciones inmunohistoquímicas resultaron positivas para la proteína S-100 en el estroma y para cromogranina y enolasa en las células ganglionares. Con estas características morfológicas, se realizó el diagnóstico de ganglioneuroma en maduración (según la clasificación de los tumores neuroblásticos del "Internacional Neuroblastoma Pathology Comité") 4 . 


\section{DISCUSIÓN}

Los tumores del grupo del neuroblastoma (tumores neuroblásticos) son tumores embrionarios del sistema nervioso simpático que derivan de la cresta neural pudiéndose originar en cualquier punto del sistema simpático paravertebral y en la médula suprarrenal. Los tumores neuroblásticos son, tras los tumores del sistema nervioso central, los tumores sólidos más frecuentes en la infancia, siendo el $15 \%$ de todas las neoplasias en los niños menores de 4 años, diagnosticándose el 90\% en menores de 5 años ${ }^{5}$.

Estos tumores son muy heterogéneos en términos de características biológicas, genéticas y morfológicas estando ampliamente documentada aunque no completamente comprendida la regresión espontánea y su maduración como fenómenos biológicos.

Clásicamente los tumores del grupo del neuroblastoma se clasificaban según criterios morfológicos en 3 tipos: Neuroblastoma, ganglioneuroblastoma y ganglioneuroma representando un espectro histológico de maduración y diferenciación. En 1999 se propuso una nueva clasificación para estos tumores, la clasificación de Shimada, recomendada por el "Internacional Neuroblastoma Pathology Comité" que relacionaba la edad con la histopatología, proporcionando diferentes valores pronósticos en diferentes estadios de la enfermedad ${ }^{4,6}$.

El ganglioneuroma es el tumor más infrecuente derivado de la cresta neural. Existe controversia sobre si los ganglioneuromas se originan de novo o por maduración o diferenciación a partir de una neuroblastoma o un ganglioneuroblastoma preexistente. Sin embargo, las diferencias tanto en edad de presentación como en la localización, apoyan la idea de que los ganglioneuromas no necesariamente se desarrollan a partir de un neuroblastoma preexistente, surgiendo la mayoría de novo Es un tumor benigno y usualmente asintomático sin existir una predilección por el sexo, pudiendo ocurrir en todos los grupos de edad, presentándose la mayoría en niños y adultos jóvenes. La localización más frecuente es en mediastino posterior y retroperitoneo, y solamente el 15-30\% de los casos notificados asientan en la glándula suprarrenal.

Microscópicamente son tumores bien circunscritos; pueden estar parcialmente rodeados por tejido fibroso, aunque no suelen tener una auténtica cáp- sula fibrosa, presentándose con una completa diferenciación. Los ganglioneuromas sintomáticos son raros y característicamente el ganglioneuroma no es un tumor secretor de catecolaminas ni de hormonas esteroideas por lo que su sintomatología derivaría de la compresión de estructuras vecinas ${ }^{7}$.

El ganglioneuroma se presenta en el TAC como una lesión sólida, homogénea con una atenuación más baja que el músculo. Algunos son heterogéneos tras la administración de contraste, pero la mayoría, tienden a permanecer menos densos que el músculo. Pueden presentar pequeñas calcificaciones hasta en un $20 \%$ de los casos, siendo una característica interesante su tendencia a envolver total o parcialmente los vasos sanguíneos sin comprometer su luz.

El manejo de los incidentalomas en adultos es principalmente quirúrgico, pudiendo ser curativo o de estadiaje. En el caso de tumores no funcionantes la decisión de exéresis quirúrgica la establece fundamentalmente el tamaño de la lesión, debiendo ser extirpada aquellas mayores de $6 \mathrm{~cm}$. Más controvertida es la actitud a seguir cuando son menores de 6 cm., ayudando e estos casos determinadas características radiológicas y gammagráficas, si bien el seguimiento clínico es otra alternativa aceptada, la mayoría de autores defienden el tratamiento quirúrgico precoz basado en grandes series y su experiencia propia. Algunos autores han indicado que incluso aquellos tumores no resecables en su totalidad podrían tener un buen pronóstico si se manejan de forma conservadora.

En la infancia, debido a la alta proporción de tumores malignos, y dado que ni la apariencia radiológica ni el tamaño tumoral ayudan a discernir entre lesiones benignas y malignas, se recomienda la resección de todos los incidentalomas suprarrenales detectados postnatalmente.

Los ganglioneuromas presentan una tendencia a la recidiva local, sobretodo si la escisión no ha sido completa, y su potencial metastático descrito en la literatura sería explicado en todo caso por su multifocalidad $^{8}$. Si no se extirpan, tras muchos años pueden malignizar en forma de Schwanomas malignos. Por tanto, se deberían realizar controles postoperatorios para detectar las recidivas locales y aparición de nuevos focos.

En caso de recidiva, debería ser considerada la cirugía como tratamiento de elección. 


\section{CONCLUSIONES}

El incidentaloma infantil es una entidad muy poco frecuente no existiendo guías clínicas para el manejo de estas masas incidentales.

En la infancia, dada la alta proporción de tumores malignos, se recomienda una actitud quirúrgica ante toda masa suprarrenal.

\section{REFERENCIAS}

1. Seddon JM, Baranetsky N, Van BOxel PJ. Adrenal "incidentalomas". Need for surgery. Urology. 1985;25(1):1-7.

2. Ciftci AO, Senocak ME, Tanyel FC, Buyukpamukcu N. Adrenocortical tumors in children. J Pediatr Surg. 2001;36(4): 549554.

3. Masiakos PT, Gerstle JT, Cheang T, Viero S, Kim PC, Wales P. Is surgery necessary for incidentally discovered adrenal masses in children? J Pediatr Surg. 2004;39(5):754-758.

4. Shimada H, Ambros IM, Dehner LP, Hata J, Joshi VV, Roald B. Terminology and morphologic criteria of neuroblastic tumors: recommendations by the International Neuroblastoma Pathology Committee. Cancer. 1999;86(2):349-363.
5. Michael Ritchey. Oncología urológica pediátrica. Patrick C. Walsh. Campbell Urología, $8^{a}$ edición. Buenos Aires, Médica Panamericana. 2004, pp 2687-2694.

6. Navarro S, Amann G, Beiske K, Cullinane CJ, d'Amore ES, Gambini $\mathrm{C}$ et al. Prognostic value of International Neuroblastoma Pathology Classification in localized resectable peripheral neuroblastic tumors: a histopathologic study of localized neuroblastoma European Study Group 94.01 Trial and Protocol. J Clin Oncol. 2006;24(4):695-699.

7. Singh KJ, Suri A, Vijjan V, Singh P, Srivastava A. Retroperitoneal ganglioneuroma presenting as right renal mass. Urology. 2006; 67(5):1085.e7-8.

8. Geoerger B, Hero B, Harms D, Grebe J, Scheidhauer K, Berthold F. Metabolic activity and clinical features of primary ganglioneuromas. Cancer. 2001;91(10):1905-1913.

Correspondencia autor: Dr. J. Arce Gil Servicio de Urología. Fundació Puigvert Cartagena, 340-350 - 08025 Barcelona. Tel. 934169700 E-mail autor: jacobo1976@hotmail.com

Información artículo: Nota clínica

Trabajo recibido: diciembre 2006 Trabajo aceptado: enero 2007 